\title{
Deconfined fractional electric charges in graphene at high magnetic fields
}

\author{
Chang-Yu Hou, ${ }^{1}$ Claudio Chamon, ${ }^{1}$ and Christopher Mudry ${ }^{2}$ \\ ${ }^{1}$ Physics Department, Boston University, Boston, Massachusetts 02215, USA \\ ${ }^{2}$ Condensed Matter Theory Group, Paul Scherrer Institut, CH-5232 Villigen PSI, Switzerland
}

(Received 28 September 2009; revised manuscript received 7 December 2009; published 23 February 2010)

\begin{abstract}
The resistance at the charge neutral (Dirac) point was shown by Checkelsky et al. [Phys. Rev. B 79, 115434 (2009)] to diverge upon the application of a strong magnetic field normal to graphene. We argue that this divergence is the signature for a Kekule instability of graphene, which is induced by the magnetic field. We show that the strong magnetic field does not remove the zero modes that bind a fraction of the electron around vortices in the Kekule dimerization pattern, and that quenched disorder present in the system makes it energetically possible to separate the fractional charges. These findings, altogether, indicate that graphene can sustain deconfined fractionalized electrons.
\end{abstract}

DOI: 10.1103/PhysRevB.81.075427

PACS number(s): 05.30.Pr, 73.20.Mf, 73.43.-f

\section{INTRODUCTION}

The elementary excitations in the fractional quantum Hall $(\mathrm{FQH})$ effect carry a fraction of the charge of the electron, as argued by Laughlin. ${ }^{1}$ Experimental evidence for the fractionally charged quasiparticles have been presented for the $\nu=1 / 3$ state, using shot-noise measurements in Refs. 2 and 3 and by using a scanning tunneling transistor in Ref. 4. It has remained an open question whether there are experimental systems in two dimensions (2D) other than the FQH states for which the elementary excitations carry a fractional charge $e^{5,6}$ and for which the fundamental mechanism for electron fractionalization is different from that in the FQH effect.

Recently, proposals for a mechanism to fractionalize the electron have been suggested for graphenelike systems, ${ }^{7}$ in which the electrons disperse according to the Dirac equation in $2 \mathrm{D}$. The mechanism involves opening a mass gap in the Dirac equation, via the spontaneous breaking of a symmetry, the formation of a Kekulé bond dimerization pattern. This mechanism is fundamentally different than the one in the FQH effect. Instead, it is closer conceptually to the mechanism for fractionalization in one dimension (1D), ${ }^{8,9}$ which for 30 years was believed to be peculiar to 1D and not possible in 2D. However, the strengths of the interactions in graphene are, alone, insufficient to lead to the Kekulé instability. Moreover, even if the Kekulé dimerization pattern formed, an axial gauge potential is needed to deconfine the fractionally charged quasiparticles that attach to vortices in the Kekulé pattern. ${ }^{10}$

We are going to show in this paper that the presence of a magnetic field in graphene stabilizes a quasi-long-range order in the form of a Kekule distortion associated to a U(1) continuous symmetry ${ }^{7,11}$ and opens an electronic energy gap $2 \Delta_{0}$. This quasi-long-range U(1) order can be destroyed by the unbinding of vortices due to either thermal- or disorderinduced fluctuations. We then show that fractional charges do attach to vortices in the Kekule pattern even in the presence of a strong magnetic field. Quenched disorder present in the system, remarkably, makes it energetically possible to separate the fractional charges. These theoretical results on the formation of the Kekule pattern in the presence of a magnetic field and of static disorder explain the features observed in the experimental measurements by Checkelsky et al. ${ }^{12,13}$

Let us briefly discuss the known phenomenology of graphene in the presence of a magnetic field. Starting from the linearized Dirac spectrum $\varepsilon(\boldsymbol{k})= \pm \hbar v_{\mathrm{F}}|\boldsymbol{k}|$ around the two nonequivalent Fermi points (valleys) $\boldsymbol{K}_{+}=-\boldsymbol{K}_{-},{ }^{14}$ the singleparticle Landau levels in a uniform applied magnetic field $B$ perpendicular to graphene are

$$
\varepsilon_{n}=\operatorname{sgn}(n) \hbar \omega_{\mathrm{c}} \sqrt{|n|} .
$$

Here, $n$ is an integer, the cyclotron frequency is $\omega_{\mathrm{c}}$ $=\sqrt{2} v_{\mathrm{F}} / \ell_{B}$, while the magnetic length is $\ell_{B}=\sqrt{\hbar c /(e B)}{ }^{15}$ This single-particle spectrum leads to the quantization of the dimensionless Hall conductivity

$$
\nu \equiv h \sigma_{x y} / e^{2}=4 \times(n+1 / 2)= \pm 2, \pm 6, \pm 10, \ldots,
$$

if the Zeeman splitting and the Coulomb or electron-phonon interactions are neglected, ${ }^{16-20}$ as observed experimentally for magnetic fields of up to $8 \mathrm{~T}^{21,22}$ On the other hand, the observation in Refs. 23 and 24 of new plateaus at the filling fractions $\nu=0, \pm 1, \pm 4$ for applied magnetic fields between 20 and $40 \mathrm{~T}$ indicates that the fourfold degeneracies of the Landau levels [Eq. (1.1)] must be lifted. For example, spin splitting may be induced by the Zeeman or Coulomb interactions while valley splitting $\left(\boldsymbol{K}_{+}\right.$and $\left.\boldsymbol{K}_{-}\right)$may be induced by Coulomb or electron-phonon interactions. Many theoretical proposals to understand these new plateaus and predict new ones have been made (for a review, see Ref. 25).

Here, we shall focus on the particularly interesting $n=0$ Landau level. The dependence of the longitudinal resistance $R_{x x}$ at this level (fixing the chemical potential $\mu=0$ ) for graphene deposited on a $\mathrm{Si}-\mathrm{SiO}_{2}$ substrate under intense uniform magnetic fields $B$ of up to $32 \mathrm{~T}$ has been measured in Refs. 12 and 13. At fixed low temperatures $(0.3-5 \mathrm{~K}), R_{x x}$ grows exponentially with magnetic fields $B>17 \mathrm{~T}$ upon approaching a sample-dependent critical field strength $B_{c}$. The critical field $B_{c}$ is larger for samples with lower zero-field mobility $\mu_{e}$. There also appears to be a scaling regime with the scaling function $R(b)=c_{1} \exp \left[2 \times c_{2}(1-b)^{-\varrho}\right] \Omega / \square$ with $c_{1}=440, c_{2}=1.54, b=B / B_{c}\left(\mu_{e}\right)<1$, and $\varrho=1 / 2$ fitting well the magnetic field dependence of $R_{x x}$ for samples character- 
ized by the mobility-dependent $B_{c}$. This scaling is reminiscent of the Kosterlitz-Thouless (KT) divergence of the correlation length in the $2 \mathrm{D}$ classical XY model. ${ }^{26}$ Finally, the temperature dependence of $R_{x x}$ for a given sample with a fixed $B$ very close to $B_{c}$ approaches the thermally activated form $\exp \left(2 \Delta_{\mathrm{a}} / T\right)$ on the interval $2 \mathrm{~K}<T<16 \mathrm{~K}^{27}$

Our proposal to explain this KT scaling is closely related to the scenario from Ref. 28. However, the disorder was treated at the level of the self-consistent Born approximation in Ref. 28, while the role of the Coulomb interaction, within the Hartree-Fock approximation, was emphasized. Here, we show that the electron-phonon interaction is critical to the selection of the Kekule instability among all 15 instabilities that can open a gap at the Dirac point while preserving the electron charge as a good quantum number. ${ }^{29}$ Moreover, we argue that a nonperturbative treatment of the disorder is also essential to explain the striking fact that $B_{c}\left(\mu_{e}\right)$ is independent of $T$ at low temperatures: it is the $2 \mathrm{D}$ classical random phase XY model that describes the phase transition observed in Ref. 13. Within this classical random phase XY model, vortices unbind when the magnetic field is below $B_{c}$, and thus deconfined fractionally charged quasiparticles can be sustained in graphene.

The line of arguments in this paper can be summarized as follows. For the relevant range of magnetic fields, the dominant energy scale originates from the kinetic energy modified by the orbital coupling to the magnetic field. The Coulomb interaction on the length scale of the magnetic length-the long-range Coulomb interaction-is the leading subdominant energy scale. To these leading and subleading orders, the dynamics of interacting electrons in graphene preserves a combined U(4) symmetry arising from the conservation of the electron charge, axial charge, ${ }^{10,29}$ and two independent spin-1/2 and valley-1/2 SU(2) symmetries. This continuous symmetry can only be broken spontaneously at zero temperature according to the Mermin-Wagner theorem. Any nonvanishing ordering temperature thus requires the explicit breaking of this $\mathrm{U}(4)$ symmetry down to products of the U(1) subgroup enforcing the conservation of the electron quantum number with either finite subgroups or U(1) subgroups of SU(4). The bare Zeeman, electron-phonon, and short-range Coulomb interactions, although close to two orders of magnitude smaller than the long-range Coulomb interaction, provide these anisotropies. In particular, the electron-phonon interaction favors the Kekulé instability over other instabilities, for example, magnetic ones, so that, even if one could turnoff electron-electron interactions, the Kekule instability would still compete with the Zeeman interaction. Hence, we first start by considering the effects of lattice distortions in the case of noninteracting electrons and without the Zeeman interaction in Sec. II. In this approximation, we show that the Kekule instability triggered by the electron-phonon coupling can explain the experiments of Ref. 13. In Sec. III, we show that as long as the Kekule energy gap in the absence of the Zeeman coupling is greater than twice the Zeeman splitting (once that is turned on), the results of Sec. II remain unchanged. However, for graphene, the value we obtain theoretically for the Kekulé gap using the electron-phonon coupling alone is too close to this threshold of twice the Zeeman splitting (as reported in Ref. 24). Therefore, a proper treat- ment of the electron-electron interactions is needed for selecting the correct low-temperature phase in Ref. 13. We restore the full Coulomb electron-electron interactions in Sec. IV. We first argue that the lattice nearest-neighbor Coulomb interaction boosts the electron-phonon coupling and stabilizes the Kekulé order in spite of the competing Zeeman splitting. We then argue that the Kekule instability and the Zeeman splitting provide the needed anisotropies to reduce the symmetry and evade the Mermin-Wagner theorem that precludes ordering at a finite temperature. The finite ordering temperature is shown to depend on both the SU(4) symmetric electron-electron interactions and on the explicit anisotropies, including those from the electron-phonon interactions. We conclude that the results obtained within the approximations of Sec. II remain valid, although the temperature scale for the transition is increased.

\section{KEKULÉ INSTABILITY}

In this section we are going to ignore the Zeeman and electron-electron interactions altogether. We will revisit this approximation in Secs. III and IV. We shall first derive the mean-field Kekulé instability induced by phonons in the presence of a magnetic field. We shall then prove the existence of zero energy states when the Kekule order supports a defect in the form of a vortex in the presence of a uniform magnetic field.

\section{A. Mean-field Kekulé order}

In the continuum limit, the orbital contribution from a magnetic field $\boldsymbol{B}=\boldsymbol{\nabla} \wedge \boldsymbol{A}$ to pristine graphene reads

$$
\mathcal{H}_{0}=\hbar v_{\mathrm{F}}\left(\boldsymbol{k}-\frac{e}{\hbar c} \boldsymbol{A}\right) \cdot \boldsymbol{\alpha},
$$

where the 2D wave number $\boldsymbol{k}$ has the components $k_{1}$ and $k_{2}$, and the Dirac matrices are

$$
\alpha_{1}=\sigma_{3} \otimes \tau_{1} \otimes s_{0}, \quad \alpha_{2}=\sigma_{3} \otimes \tau_{2} \otimes s_{0} .
$$

The unit $2 \times 2$ matrices $\sigma_{0}, \tau_{0}$, and $s_{0}$ together with the Pauli matrices $\boldsymbol{\sigma}, \boldsymbol{\tau}$, and $\boldsymbol{s}$ act on the valley-1/2 $\left(\boldsymbol{K}_{+}\right.$and $\left.\boldsymbol{K}_{-}\right)$, sublattice-1/2 (A and B), and spin-1/2 ( $\uparrow$ and $\downarrow$ ) twodimensional subspaces of graphene, respectively.

The spectrum of $\mathcal{H}_{0}$ is fourfold degenerate, for $\mathcal{H}_{0}$ commutes with the 16 Hermitean generators

$$
\begin{aligned}
& X_{00 \kappa}:=\sigma_{0} \otimes \tau_{0} \otimes s_{\kappa}, \quad X_{13 \kappa}:=\sigma_{1} \otimes \tau_{3} \otimes s_{\kappa}, \\
& X_{23 \kappa}:=\sigma_{2} \otimes \tau_{3} \otimes s_{\kappa}, \quad X_{30 \kappa}:=\sigma_{3} \otimes \tau_{0} \otimes s_{\kappa}
\end{aligned}
$$

(here $\kappa=0,1,2,3$ ) of the Lie group

$$
\mathrm{U}(4)=\mathrm{U}(1) \times \mathrm{SU}(4) \simeq \mathrm{SO}(6) .
$$

Notice the electron charge U(1) subgroup generated by

$$
X_{000} \text {, }
$$

the spin-1/2 SU(2) subgroup generated by

$$
X_{001}, \quad X_{002}, \quad X_{003},
$$

and the valley-1/2 SU(2) subgroup generated by 


$$
X_{130}, \quad X_{230}, \quad X_{300} \equiv \gamma_{5} .
$$

A Kekule instability is a periodic modulation of the nearest-neighbor hopping amplitude in graphene with the wave vector $\boldsymbol{K}_{+}-\boldsymbol{K}_{-}{ }^{7}$ In the continuum approximation Eq. (2.1), it is represented by the Hermitean mass matrix

$$
\mathcal{M}_{\mathrm{K}}:=\boldsymbol{\Delta} \cdot \boldsymbol{M} \equiv \Delta_{1} M_{1}+\Delta_{2} M_{2}
$$

that is parametrized by the real-valued numbers $\Delta_{1}$ and $\Delta_{2}{ }^{7}$ The Kekulé mass matrices are

$$
M_{1}:=\sigma_{1} \otimes \tau_{0} \otimes s_{0}, \quad M_{2}:=-\sigma_{2} \otimes \tau_{0} \otimes s_{0},
$$

where a mass matrix is any Hermitean matrix

$$
X_{\kappa_{1} \kappa_{2} \kappa_{3}}:=\sigma_{\kappa_{1}} \otimes \tau_{\kappa_{2}} \otimes s_{\kappa_{3}}, \quad \kappa_{1}, \kappa_{2}, \kappa_{3}=0,1,2,3
$$

that anticommutes with $\mathcal{H}_{0}$. [There are 16 mass matrices as shown in Ref. 29. They generate a group U(4) distinct from the group $\mathrm{U}(4)$ whose generators are given by Eq. (2.2).] In the continuum approximation, only the eight generators

$$
X_{00 \kappa}, \quad \Delta_{1} X_{13 \kappa}-\Delta_{2} X_{23 \kappa},
$$

(where $\kappa=0,1,2,3$ ) of the Lie group [Eq. (2.2)] commute with

$$
\mathcal{H}:=\mathcal{H}_{0}+\mathcal{M}_{\mathrm{K}} .
$$

In the presence of the uniform magnetic field $B=\partial_{1} A_{2}-\partial_{2} A_{1}$ and the uniform Kekule order parameter $\Delta=\Delta_{1}+i \Delta_{2}=\Delta_{0} e^{i \theta_{0}}$, for $0 \leq \Delta_{0}$ and $0 \leq \theta_{0}<2 \pi$ the singleparticle spectrum is the shifted Landau spectrum

$$
\varepsilon_{N, \pm}= \pm \sqrt{\left(\hbar \omega_{\mathrm{c}}\right)^{2} N+\Delta_{0}^{2}},
$$

where $N=0,1,2, \ldots$

The mean-field value of the Kekulé gap $2 \Delta_{0}$ induced by a magnetic field and by an electron-phonon coupling in the single-particle approximation (see also Ref. 30) is obtained by balancing the gains in the electronic energy against the losses in the elastic energy of the lattice

$$
\delta E \equiv \delta E_{\text {elec }}+\delta E_{\text {pho }},
$$

where

$$
\begin{aligned}
\delta E_{\text {elec }} & =2 \times \frac{\mathcal{A}}{2 \pi \ell_{B}^{2}} \sum_{N=0}^{\infty}\left(2-\delta_{0, N}\right)\left(\varepsilon_{N,-}-\varepsilon_{-N}\right) \\
& =-2 \times \frac{\mathcal{A}}{2 \pi \ell_{B}^{2}} \Delta_{0}+\mathcal{O}\left(\frac{\Delta_{0}^{2}}{\hbar \omega_{\mathrm{c}}}\right)
\end{aligned}
$$

is the electronic gain in energy, while

$$
\delta E_{\mathrm{pho}}=\frac{\mathcal{N}}{2} K_{\Delta} u_{0}^{2}=\frac{\mathcal{N}}{2} \frac{K_{\Delta}}{f_{\Delta}^{2}} \Delta_{0}^{2}
$$

is the cost in elastic energy. Here, $K_{\Delta}$ is the bond elastic constant and $f_{\Delta}$ ties the gap $2 \Delta_{0}$ to the atomic displacement $u_{0}$ for the Kekulé order, which results because of the change in hopping matrix elements from the orbital overlaps. [The contribution to the electronic gain from the levels $N=1,2, \ldots$ can be absorbed into a downward renormalization of the elastic rigidity $K_{\Delta} / f_{\Delta}^{2}$ (Ref. 31).] We have as- sumed that the cyclotron energy is the largest energy scale of the problem. We have also introduced the area $\mathcal{A}$ and the number $\mathcal{N}=4 \mathcal{A} /\left(3 \sqrt{3} \mathfrak{a}^{2}\right)$ of sites of graphene, $\mathfrak{a} \approx 1.42 \AA$ is the lattice spacing. The absolute minimum of Eq. (2.10) is obtained for the mean-field value

$$
\Delta_{0}=\frac{f_{\Delta}^{2}}{\mathcal{N} K_{\Delta}} \frac{\mathcal{A}}{\pi \ell_{B}^{2}}=\frac{3 \sqrt{3} \mathfrak{a}^{2}}{4 \pi \ell_{B}^{2}} \frac{f_{\Delta}^{2}}{K_{\Delta}}
$$

of the Kekule single-particle mass. If, following Ref. 30, we make the estimate $f_{\Delta}^{2} / K_{\Delta} \approx 6.366 \mathrm{eV}$, then the mean-field Kekulé gap is estimated to be

$$
2 \times \Delta_{0} \approx 1.86 \times B[\mathrm{~K}],
$$

in units of kelvin (with $B$ measured in units of the tesla). The single-particle electronic gap Eq. (2.12) overestimates by a factor of $\approx 2$ the measured activation gaps. ${ }^{13,27}$

We now discuss the all important phase fluctuations of the Kekulé order parameter and the effective action that governs them. The complex-valued Kekulé order parameter $\Delta=\Delta_{0} e^{i \theta}$ has a phase $\theta(\boldsymbol{r}, t)$ that can fluctuate in space and in time. The dependence of the effective action on this phase stems from the dynamics of the phonons on the one hand and from integrating out the electronic degrees of freedom on the other hand.

In addition, we shall also account for the fluctuations in the hopping matrix elements due to disorder or to local curvature effects (tiny ripples) on the surface of graphene,

$$
\delta \mathcal{H}=-\hbar v_{\mathrm{F}} \boldsymbol{A}_{5} \cdot \gamma_{5} \boldsymbol{\alpha} .
$$

The axial vector potential $\boldsymbol{A}_{5}$ encodes these disorder effects in the hopping matrix elements. Notice the

$$
\gamma_{5}=\sigma_{3} \otimes \tau_{0} \otimes s_{0}
$$

matrix, so that $\boldsymbol{A}_{5}$ couples with opposite signs to the two flavors at $\boldsymbol{K}_{+}$and $\boldsymbol{K}_{-}$, in contrast to the electromagnetic vector potential $\boldsymbol{A}$.

As shown by Jackiw and $\mathrm{Pi},{ }^{10}$ this axial vector potential is intimately related to the phase of the Kekule order parameter. The axial gauge transformation $\Psi \rightarrow e^{i \gamma_{5} \theta / 2} \Psi$ on the singleparticle spinor $\Psi$ for electrons, removes the phase of the Kekulé order parameter, $\Delta \rightarrow \Delta e^{-i \theta}=\Delta_{0}$, by shifting $A_{5 \mu} \rightarrow A_{5 \mu}-\partial_{\mu} \theta / 2$ (where $\mu=0,1,2$ ). The effective action that follows upon integrating the electrons can be inferred from symmetry. ${ }^{29}$ The leading term is, at $T=0$, $\left[\partial_{\mu} \equiv\left(v_{\mathrm{F}}^{-1} \partial_{t}, \partial_{r}\right)\right]$

$$
S=\frac{J}{2 \hbar} \int d t d^{2} r\left(\partial_{\mu} \theta-2 A_{5 \mu}\right)^{2} .
$$

The stiffness $J$ was computed at zero magnetic field in Ref. 29. Now, we compute $J$ in the presence of $B$. To this end, we consider the time-independent Kekule texture with $\theta=\boldsymbol{q} \cdot \boldsymbol{r}$ and $\boldsymbol{A}_{5}=0$, or the gauge equivalent $\theta=0$ and $\boldsymbol{A}_{5}=-\boldsymbol{q} / 2$. We solve for the eigenvalues of $\mathcal{H}+\delta \mathcal{H}$ treating $\boldsymbol{A}_{5}=-\boldsymbol{q} / 2$ to second order in perturbation theory (see details in Appendix A). The electronic energy cost of $\boldsymbol{A}_{5}=-\boldsymbol{q} / 2$ to order $|\boldsymbol{q}|^{2} \mathrm{de}$ termines the stiffness 


$$
J=\frac{\Delta_{0}}{2 \pi} .
$$

(This result coincides with the stiffness computed at zero field in Ref. 29, once spin degeneracy is accounted there).

We shall treat the $\boldsymbol{A}_{5}$ in Eq. (2.15) as a static random phase on phenomenological grounds dictated by symmetry. A microscopic justification for this step is the following. In a realistic sample, the matrix hopping elements between nearest-neighbor atoms will not be all the same, but there will be fluctuations caused by several factors, strain when in contact with the substrate being one factor. The random hopping amplitudes not only cause the phase in Eq. (2.15) to be random but also the coupling $J$ to be spatially varying. However, weak random exchange is irrelevant, and we thus simply focus on the uniform $J$ case.

The effective action on length scales larger than $\ell_{B}$ is thus that of the quantum random phase $\mathrm{XY}$ model in $2 \mathrm{D}$ whereby we assume the white-noise distribution of vanishing mean (overline denotes disorder averaging)

$$
\overline{A_{5 i}(\boldsymbol{r}) A_{5 j}\left(\boldsymbol{r}^{\prime}\right)}=g \delta_{i j} \delta^{(2)}\left(\boldsymbol{r}-\boldsymbol{r}^{\prime}\right), \quad i, j=1,2 .
$$

It is crucial to appreciate that $g$ is a dimensionless coupling constant. It thus depends on the magnetic field through a function $g\left(\ell / \mathfrak{a}, \ell_{B} / \ell\right)$ of two arguments. The argument $\ell / \mathfrak{a}$ is the mean free path associated with the disorder for the system at $B=0$ in units of the lattice spacing $\mathfrak{a}$. The argument $\ell_{B} / \ell$ must also be present because as $\ell_{B}$ decreases with increasing $B$, the single-particle overlaps due to disorder of (otherwise orthogonal) orbitals decrease.

Next, we are going to argue that the 2D random phase $\mathrm{XY}$ model defined by Eqs. (2.15)-(2.17) solves two experimental puzzles observed in Ref. 13. Experimentally, the resistivity of graphene is fit to a form consistent with the scaling of the correlation length $\xi$ of the 2D classical XY model, where the distance to the critical point is measured in terms of the reduced field $b=B / B_{c}$. Why is it that the data fits a transition as a function of $b$ in the 2D universality class of the classical as opposed to the quantum XY model? Why is it that the resistivities are rather temperature independent for temperatures differing by a factor of 5 , from $0.3 \mathrm{~K}$ to $1.5 \mathrm{~K}$ ?

First, the measured KT scaling could be explained if the fluctuations driving the transition were 1D but of quantum origin. For instance, if the transition had to do with edge physics. This idea has been proposed in Ref. 32. The problem with this scenario is that it requires (1) a large Zeeman coupling, (2) a critical density of magnetic impurities, and (3) the ordered phase in a 1D quantum system does not survive finite temperatures so that the size of the region in which the correlation length scales in a KT fashion near the quantum critical point is bounded from above by an exponentially small crossover temperature.

Second, a quantum critical point of the $2 \mathrm{D}$ quantum $\mathrm{XY}$ model [Eq. (2.15)], without static random phases, does not obey the KT scaling that fits the data in Ref. 13 over three decades of values of longitudinal resistances $R(b)$. On the other hand, a KT scaling is compatible with the clean limit of model [Eq. (2.15)] if the relevant range of temperatures for which quasi-long-range order holds is large enough so that

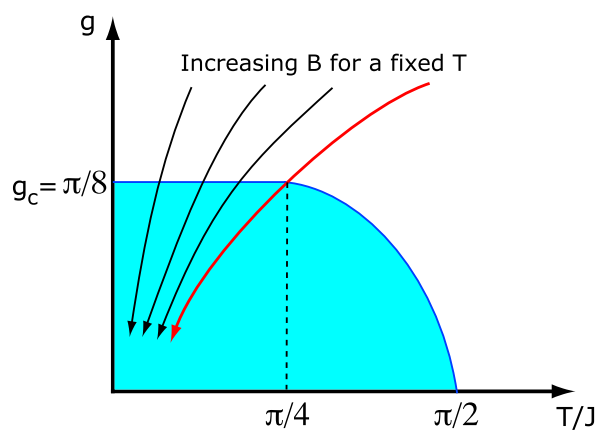

FIG. 1. (Color online) Phase diagram of the 2D classical random phase XY model after Ref. 34. The horizontal axis represents the temperature $T$ in units of the stiffness $J$. The vertical axis represents the dimensionless disorder strength $g$ (the variance for white-noise and Gaussian correlated random phases). The shaded region depicts the quasi-long-range ordered phase for which the interaction between vortices is logarithmic. The complementary white region depicts the paramagnetic phase for which the interaction between vortices is screened beyond the screening length $\xi$. At the transition line that separates the two phases, vortices undergo a confiningdeconfining transition. We interpret the divergence of the resistance of graphene subjected to a strong increasing magnetic field $B$ measured in Ref. 13 as being governed by the increase of the screening length $\xi(B)$ in the paramagnetic region along a RG trajectory parametrized by $B$. Here, $T / J$ and $g$ are both decreasing functions of $B$.

quantum critical fluctuations can be neglected (see Ref. 33). However, even if the quantum critical fluctuations can be neglected when the temperature changes from 0.3 to $1.5 \mathrm{~K}$, the position of the critical field $B_{c}$ should move with temperature, for the location of the clean KT transition is where $J(B)=2 T / \pi$.

Third, the disorder in Eq. (2.15) can explain the observed $\mathrm{KT}$ scaling. Indeed, the 2D classical random phase XY model has the remarkable feature that its boundary separating the quasi-long-range ordered phase from the paramagnetic phase runs (nearly) parallel to the temperature axis when $T<T_{\mathrm{KT}} / 2$, where $T_{\mathrm{KT}}=\pi / 2 J{ }^{34}$ (This feature is nonperturbative in disorder. ${ }^{35,36}$ ) However, no consensus has emerged on the exponent $\varrho$ that characterizes the diverging correlation length $\exp \left(b / x^{\varrho}\right)$ upon approaching the boundary (it is debated if it is $\varrho=1 / 2$ or 1 ). The phase diagram is shown in Fig. 1. Also shown are the trajectories of coordinates $\left[T / J(B), g\left(\ell / \mathfrak{a}, \ell_{B} / \ell\right)\right]$ as the magnetic field $B$ is changed for fixed $\ell$ and $T$. When these trajectories enter the quasi-long-ordered phase through the disorder-controlled boundary parallel to the $T / J$ axis, one has the condition

$$
g\left(\ell / \mathfrak{a}, \ell_{B} / \ell\right)=\pi / 8,
$$

i.e.,

$$
B=B_{c}(\mathfrak{a}, \ell / \mathfrak{a})
$$

which is temperature independent as long as

$$
T<T_{\mathrm{KT}} / 2,
$$

i.e., 


$$
T<\Delta_{0}\left(B_{c}\right) / 8 .
$$

One can estimate the upper range of temperatures for which the transition is KT-like as a function of the scaling parameter $b=B / B_{c}$ used in Ref. 13. For $B_{c} \approx 29.1 \mathrm{~T}$, one finds using Eqs. (2.12) and (2.18b) that the $2 \mathrm{D}$ classical scaling as a function of $b$ holds as long as $T<3.5 \mathrm{~K}$.

These results thus explain rather well the features observed in Ref. 13. Accordingly, the Kekule distortion, which does not appear to occur in graphene at zero magnetic field, is induced by the presence of the magnetic field above a critical field $B_{c}$ that depends on the disorder strength and is thus sample dependent.

\section{B. Zero modes in a uniform magnetic field}

One of the surprising features in graphene is that vortices in the Kekule dimerization pattern bind fractionalized excitations when $B=0 .^{7}$ We now show analytically that fractionalization is robust to the presence of $B$, i.e., there is a zero mode due to vortices that binds a fraction of the electron for any value of $B$.

We thus seek $\varepsilon=0$ solutions of the Dirac operator [Eq. (2.8)] when the Kekulé order parameter supports the vortex

$$
\Delta(\boldsymbol{r}) \equiv \Delta(\rho, \theta)=\Delta_{0} e^{i \theta_{0}} e^{i n_{\mathrm{v}} \theta} .
$$

To this end, we use polar coordinates and the symmetric gauge $\boldsymbol{A}=B(-y, x) / 2$. Without loss of generality, we restrict the vorticities to $n_{\mathrm{v}}= \pm 1$. It follows from the sublattice symmetry in the problem that, as in the case when $B=0$, the zero-mode solutions have support on either the $A$ (for $n_{\mathrm{v}}=-1$ ) or B sublattice (for $n_{\mathrm{v}}=+1$ ). The solutions in the presence of the magnetic field (see details in Appendix B) are

$$
\Psi_{\mathrm{A}}(\boldsymbol{r})=\left(\begin{array}{c}
0 \\
u(\rho) \\
v(\rho) \\
0
\end{array}\right), \quad \Psi_{\mathrm{B}}(\boldsymbol{r})=\left(\begin{array}{c}
v(\rho) \\
0 \\
0 \\
-u(\rho)
\end{array}\right),
$$

where

$$
\begin{gathered}
u(\rho)=i e^{-i \theta_{0} / 2} \sqrt{p} D_{-p-1}\left(\rho / \ell_{B}\right), \\
v(\rho)=e^{+i \theta_{0} / 2} D_{-p}\left(\rho / \ell_{B}\right),
\end{gathered}
$$

$D_{p}(z)$ is the parabolic cylinder function, ${ }^{37}$ and the dimensionless ratio

$$
p=\frac{2 \Delta_{0}^{2}}{\left(\hbar \omega_{\mathrm{c}}\right)^{2}} .
$$

The existence of a single midgap state for a vortex $\left(n_{\mathrm{v}}=+1\right)$ or antivortex $\left(n_{\mathrm{v}}=-1\right)$ implies that these topological defects bind half of the electron charge $\pm 1 / 2$. $^{7}$ We have verified numerically (see Fig. 2) that the effect of breaking the sublattice symmetry with a staggered chemical potential $\mu_{\mathrm{s}}$ (a coexisting charge-density wave) is to move the midgap states away from $\mu=0$. According to Fig. 2, the fractional charge bound to a vortex acquires a dependence on the value

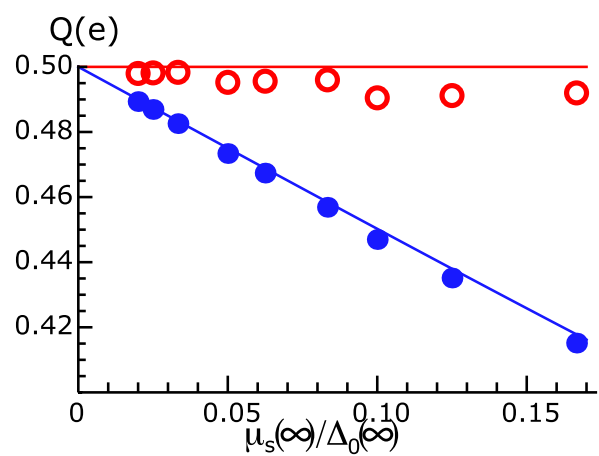

FIG. 2. (Color online) Fractionalized charge $Q$ in units of the electric charge $e$ of electrons in the presence of a magnetic field $B=20 \mathrm{~T}$ as a function of the ratio between the staggered chemical potential $\mu_{\mathrm{s}}(\infty)$ and the Kekule gap $\Delta_{0}(\infty)$ very far away from a single vortex (filled circles) or very far away from a pair of topological defects made of a vortex and a half axial flux (open circles). The lattice model consists of $113 \times 80$ sites. The solid lines are the analytical results presented in Ref. 29 when $B=0$.

of $\mu_{\mathrm{s}}$. Remarkably, we can fit this dependence in the presence of the strong magnetic field $B$ with the results obtained in Refs. 7, 10, 29, 38, and 39 in the absence of the magnetic field! Altogether, our analytical and numerical results show that the fractionalization of the electric charge of electrons is robust to any magnetic field.

\section{COMPETING ZEEMAN ENERGY}

So far, we have ignored the Zeeman energy. The reason to do so is twofold. First, the Kekule order parameter is a spin singlet and thus all preceding considerations apply in the presence of a Zeeman interaction, if it can be established that the Kekule instability survives the presence of the Zeeman interaction. Second, as we show below, the mean-field Kekulé instability Eq. (2.11) is unchanged by the addition to $\mathcal{H}$ defined in Eq. (2.8) of the Zeeman interaction

$$
\mathcal{H}_{\mathrm{Z}}:=\Delta_{\mathrm{Z}} \sigma_{0} \otimes \tau_{0} \otimes s_{3}
$$

if

$$
0 \leq \Delta_{\mathrm{Z}}<\frac{\Delta_{0}}{2},
$$

while there is no Kekule instability if

$$
0 \leq \frac{\Delta_{0}}{2}<\Delta_{\mathrm{Z}}
$$

the case of

$$
\frac{\Delta_{0}}{2}=\Delta_{\mathrm{Z}}
$$

being a mean-field first-order transition. Hence, if condition in Eq. (3.2) holds, it is legitimate to ignore the Zeeman energy altogether when computing the magnitude of the Kekulé gap.

This is not to say that the Zeeman interaction does not play an essential role, for it is it together with the Kekule 
order parameter that establishes the desired pattern of explicit symmetry breaking

$$
\begin{gathered}
\mathcal{H}_{0} \rightarrow \mathcal{H}_{0}+\mathcal{H}_{\mathrm{K}}+\mathcal{H}_{\mathrm{Z}}, \\
\mathrm{U}(4) \rightarrow \mathrm{U}(1) \times \mathrm{U}(1) \times \mathrm{U}(1) \times \mathrm{U}(1)
\end{gathered}
$$

with the residual unbroken $\mathrm{U}(1)$ symmetry generators

$$
X_{000}, \quad X_{003}, \quad \Delta_{1} X_{130}-\Delta_{2} X_{230}, \quad \Delta_{1} X_{133}-\Delta_{2} X_{233},
$$

respectively.

To understand results in Eqs. (3.2)-(3.4), it suffices to observe that the presence of the Zeeman interaction Eq. (3.1) changes the single-particle spectrum Eq. (2.9) to

$$
\varepsilon_{N, \pm} \rightarrow\left\{\begin{array}{l}
+\sqrt{\left(\hbar \omega_{\mathrm{c}}\right)^{2} N+\Delta_{0}^{2}}+\Delta_{\mathrm{Z}}, \\
+\sqrt{\left(\hbar \omega_{\mathrm{c}}\right)^{2} N+\Delta_{0}^{2}}-\Delta_{\mathrm{Z}}, \\
-\sqrt{\left(\hbar \omega_{\mathrm{c}}\right)^{2} N+\Delta_{0}^{2}}+\Delta_{\mathrm{Z}}, \\
-\sqrt{\left(\hbar \omega_{\mathrm{c}}\right)^{2} N+\Delta_{0}^{2}}-\Delta_{\mathrm{Z}},
\end{array}\right.
$$

where $N=0,1,2, \ldots$ It then follows that the gain in the kinetic energy Eq. (2.10b) is changed to

$$
\begin{aligned}
\delta E_{\text {elec }} \rightarrow & -2 \times \frac{\mathcal{A}}{2 \pi \ell_{B}^{2}} \Theta\left(\Delta_{0}-\Delta_{\mathrm{Z}}\right)\left(\Delta_{0}-\Delta_{\mathrm{Z}}\right) \\
& +4 \times \frac{\mathcal{A}}{2 \pi \ell_{B}^{2}} \sum_{N=1}^{\infty}\left(\varepsilon_{N,-}-\varepsilon_{-N}\right)
\end{aligned}
$$

where $\Theta$ is the Heaviside function. The difference between the total (electronic and phonon) energy with and without the Kekulé order is

$$
\delta E=-2 \times \frac{\mathcal{A}}{2 \pi \ell_{B}^{2}} \Theta\left(\Delta_{0}-\Delta_{\mathrm{Z}}\right)\left(\Delta_{0}-\Delta_{\mathrm{Z}}\right)+\frac{\mathcal{N}}{2} \frac{K_{\Delta}}{f_{\Delta}^{2}} \Delta_{0}^{2}
$$

according to Eq. (2.10c). Condition in Eq. (3.2) follows from seeking the global minimum of $\delta E\left(\Delta_{0}\right)$ with $\Delta_{0}>0$ and $\delta E\left(\Delta_{0}\right)<0$.

The measurements from Jiang et al. in Ref. 24 give the estimate

$$
2 \times \Delta_{\mathrm{Z}} \approx 1.3 \times B[\mathrm{~K}]
$$

for the Zeeman splitting. Hence, although the phononinduced Kekulé gap [Eq. (2.12)] is larger that the one measured in Ref. 24, it is short of satisfying condition in Eq. (3.2), unless it is enhanced by another mechanism, say by the short-range part of the Coulomb electron-electron repulsions. Indeed, there are contributions other than phonons that also favor the Kekule instability. For example, in Ref. 7, the nearest-neighbor (extended Hubbard) potential was found to favor the Kekulé instability for strong enough coupling. In the next section, we turn to the discussion of the Coulomb interaction, and the issues of its symmetries and anisotropies. We will show that the anisotropies are essential to lower the symmetry of the problem to either a discrete symmetry or an abelian U(1) symmetry, so that ordering can take place in the
2D system. The fact that a Kosterlitz-Thouless transition is seen in the experiments of Ref. 12 suggests that the interactions are sufficient to boost the U(1) Kekule ordering over the Zeeman term.

\section{ELECTRON-ELECTRON INTERACTIONS}

We have ignored Coulomb electron-electron repulsions so far. In the absence of magnetic fields and by power counting, short-range interactions are marginally irrelevant while the three-dimensional (3D) long-range Coulomb interaction is marginal. In the latter case, it is an empirical fact that the Coulomb long-range interaction is marginally irrelevant in the samples of graphene studied to this date.

The 3D long-range Coulomb interaction takes the form

$$
\hat{H}_{\mathrm{Cb}}=\frac{1}{2} \int d^{2} \boldsymbol{r} \int d^{2} \boldsymbol{r}^{\prime} \hat{\rho}(\boldsymbol{r}) V_{\mathrm{Cb}}(\boldsymbol{r}) \hat{\rho}\left(\boldsymbol{r}^{\prime}\right) .
$$

The local electronic density operator

$$
\hat{\rho}(\boldsymbol{r}):=\left(\hat{\psi}^{\dagger} \hat{\psi}\right)(\boldsymbol{r})
$$

is constructed from the eight-component spinor-valued operators $\hat{\psi}^{\dagger}(\boldsymbol{r})$ and $\psi(\boldsymbol{r})$ that follow upon second quantization of the Dirac single-particle $8 \times 8$ Hamiltonian (2.1)

$$
\hat{H}_{0}:=\int d^{2} \boldsymbol{r} \hat{\psi}^{\dagger}(\boldsymbol{r}) \mathcal{H}_{0}(\boldsymbol{r}) \hat{\psi}(\boldsymbol{r}) .
$$

Finally, the 3D Coulomb two-body repulsive interaction is

$$
V_{\mathrm{Cb}}(\boldsymbol{r})=\frac{e^{2}}{\epsilon|\boldsymbol{r}|}
$$

with $\epsilon$ the effective dielectric constant resulting from the substrate below and the air above graphene.

The long-range Coulomb interaction Eq. (4.1) shares the global U(8) symmetry of the coarse-grain electronic density Eq. (4.2) under

$$
\hat{\psi}^{\dagger} \rightarrow \hat{\psi}^{\dagger} \mathcal{U}^{\dagger}, \quad \hat{\psi} \rightarrow \mathcal{U} \hat{\psi}, \quad \mathcal{U} \in \mathrm{U}(8) .
$$

This global U(8) symmetry is broken down to its $U(4)$ subgroup generated by the generators in Eqs. (2.2a) and (2.2b) that leave the kinetic energy in Eq. (4.3) invariant.

The characteristic energy scale of the long-range Coulomb interaction in the presence of a magnetic field is estimated in Refs. 40-42 to be

$$
120 \sqrt{B}[\mathrm{~K}] \sim \frac{e^{2}}{\epsilon \ell_{B}}<\hbar \omega_{\mathrm{c}} \sim 400 \sqrt{B}[\mathrm{~K}]
$$

in units of kelvin (with $B$ measured in units of the tesla). This makes the long-range Coulomb interaction the second largest energy scale for the range of magnetic fields relevant to Ref. 13 after that arising from the kinetic energy Eq. (4.3).

The U(8) symmetry Eq. (4.5) of the long-range Coulomb interaction is not exact. On the length scale of the honeycomb lattice spacing, the local electronic density operator is not given by the coarse-grain approximation [Eq. (4.2)] but it resolves the sublattice- $1 / 2$ and valley-1/2 quantum numbers 
down to the point-group symmetry of the tight-binding model. Consequently, the on-site or the nearest-neighbor density-density repulsive interactions break the U(8) symmetry down to the product between the $\mathrm{U}(2)$ subgroup that generates the conservation of the charge and of the spin-1/2 quantum numbers on the one hand and of the discrete point group on the other hand. The spin-1/2 SU(2) symmetry is, in turn, broken by the Zeeman interaction Eq. (3.1).

The characteristic energy scale for the Coulomb repulsion on the scale of the lattice spacing must be of the order

$$
\frac{e^{2}}{\ell_{B}} \times f\left(\mathfrak{a} / \ell_{B}\right)
$$

with $f$ a dimensionless function obeying $f(x \rightarrow 0)=0$. If the asymptotic expansion $f(x \rightarrow 0) \sim x$ holds, it then follows that the characteristic energy scale of the lattice Coulomb interaction grows linearly with $B$

$$
\frac{e^{2}}{\ell_{B}} \times f\left(\mathfrak{a} / \ell_{B}\right) \sim B
$$

and could thus be comparable to the Zeeman splitting [Eq. (3.9)] or to the phonon-induced Kekulé gap Eq. (2.12). We shall assume this to be the case.

Because of the Mermin-Wagner theorem, the long-range Coulomb interaction cannot break spontaneously the combined U(4) spin and valley symmetries of the kinetic energy $\mathcal{H}_{0}$ from Eq. (4.3) at any nonvanishing temperature. However, any subdominant interaction that breaks the U(4) symmetry down to a $\mathrm{U}(1)$ or a finite subgroup of $\mathrm{U}(4)$ (say its center $\left.\mathbb{Z}_{4}\right)$ can establish a nonvanishing-temperature transition to a quasi-long-range or long-range-order phase, respectively. Hence, any of the Zeeman, the phonon-electron, or the short-range Coulomb interactions can induce a nonvanishing-temperature phase transition. This brings about two questions. What is the leading instability and at what temperature does the leading instability takes place?

First, since any finite-temperature instability must be associated to a discrete symmetry or to a U(1) symmetry subgroup of U(4), the empirical observation of a KT-like transition rules out any discrete symmetry subgroup. This empirical fact can be understood at the mean-field level from three observations. (i) We showed in Secs. II and III that phonons favor a Kekulé instability with a gap larger (but not substantially larger) than the Zeeman splitting. (ii) Moreover, a repulsive nearest-neighbor Coulomb interaction reinforces the phonon-induced instability. ${ }^{7}$ (iii) An exhaustive list of instabilities in graphene can be found in Ref. 29. Because the cyclotron energy is the dominant energy scale for the relevant range of magnetic field, we can ignore U(1) instabilities associated to superconductivity. This still leaves several competing U(1) order parameters (see Table 1 in Ref. 29) but of these only one, the Kekule instability, is also favored by phonons.

Second, we have seen in Eq. (3.6) that the Zeeman interaction $\mathcal{H}_{\mathrm{Z}}$ opens a single-particle gap at half-filling in the Landau level $\nu=0$, i.e., the Slater determinant made of all $\nu=0$ single-particle energy eigenstates of $\mathcal{H}_{0}$ in Eq. (2.1) with negative eigenenergies has a larger energy than that of
$\mathcal{H}_{0}+\mathcal{H}_{\mathrm{Z}}$ at half-filling. The Zeeman term is also a symmetrybreaking field that lowers the U(4) symmetry of $\mathcal{H}_{0}$ in Eq. (2.1). Any of the 15 traceless generators of the U(4) symmetry [Eq. (2.2)] would do the same, or, more generally any 15-component uniform real-valued "SU(4)-symmetrybreaking magnetic field" $b=\left(b_{\mathrm{a}}\right)$ in

$$
\mathcal{H}(b):=\sum_{\mathrm{a}=1}^{15} b_{\mathrm{a}} T_{\mathrm{a}},
$$

$$
T_{1}:=\frac{1}{\sqrt{16}} X_{001}, \ldots, \quad T_{15}:=\frac{1}{\sqrt{16}} X_{303},
$$

opens a single-particle gap if added to $\mathcal{H}_{0}$. [The factor of $1 / \sqrt{16}$ insures the normalization $\operatorname{tr} T_{\mathrm{a}} T_{\mathrm{b}}=\delta_{\mathrm{ab}} / 2$ for the 15 generators $T_{\mathrm{a}}$ of $\mathrm{SU}(4)$.]

These single-particle considerations are also valid in the presence of the Coulomb interaction. As is the case in double-layer nonrelativistic quantum Hall systems, ${ }^{43-45}$ the long-range Coulomb interaction in the $\nu=0$ Landau level is minimized by breaking spontaneously the SU(4) symmetry in Eq. (2.2) in the same way as a quantum SU(4) ferromagnet would do, ${ }^{40-42}$ i.e., in such a way that $\hat{\psi}^{\dagger}(\boldsymbol{r}) \mathcal{H}(b) \hat{\psi}(\boldsymbol{r})$ acquires spontaneously a finite expectation value independent of $\boldsymbol{r}$ in the many-body ground state for some "SU(4)symmetry-breaking magnetic field" $b=\left(b_{\mathrm{a}}\right)$. Such a manybody ground state is symmetric under any exchange of a pair of its SU(4) indices. Consequently, the Pauli principle can maximize the spatial separation of electrons to lower the Coulomb interaction without penalizing the kinetic energy due to the magnetic field.

If the Coulomb interaction is treated in a mean-field approximation by which $\hat{H}_{\mathrm{Cb}} \rightarrow \mathcal{H}(b)$ for some "SU(4)symmetry-breaking magnetic field" $b=\left(b_{\mathrm{a}}\right)$, the presence of a single-particle mean-field gap justifies integrating the fermions in a gradient expansion. If this is done at any small but nonvanishing temperature, we can ignore quantum fluctuations to a first approximation. There follows an effective classical theory that describes how small thermal fluctuations about the SU(4)-symmetry-broken ground state restore the full SU(4) symmetry, as dictated by the Mermin-Wagner theorem (we always assume that the dominant cyclotron energy prevents any superconducting instability). On symmetry grounds, this classical theory is a nonlinear-sigma model (NLSM) on the target manifold $G / H$ given by the $N$-1-dimensional complex projective space

$$
\begin{aligned}
\mathrm{CP}^{N-1} & \simeq \mathrm{U}(N) / \mathrm{U}(N-1) \times \mathrm{U}(1) \\
& \simeq \mathrm{SU}(N) / S(\mathrm{U}(N-1) \times \mathrm{U}(1))
\end{aligned}
$$

with $N=4$ (see Refs. 46-49 for the introduction and overview of $\mathrm{CP}^{N-1} \mathrm{NLSM}$ ). The little group $H$ is here the largest subgroup of $G$ that leaves the ground state $\psi(b)$ of $\mathcal{H}(b)$ invariant. It is made of the direct product of the subgroup SU(3) spanned by all unitary transformation in the subspace orthogonal to the direction of $\psi(b)$ in the combined spin and valley subspace and the subgroup $U(1)$ spanned by all rotations about $\psi(b)$ in the very same spin and valley subspace. If we also include the symmetry-breaking subleading inter- 
actions (the Zeeman, electron-phonon, and electron-electron short-range Coulomb interactions), this effective theory depends on two-dimensionless couplings. There is the reduced temperature $t$. There also is the coupling $\lambda \ll t$ that breaks the SU(4) symmetry to subleading order so as to enforce the desired pattern [Eq. (3.5)] of explicit symmetry breaking. By power counting, $t$ is marginal while $\lambda$ is strongly relevant. (These couplings have already been renormalized by quantum fluctuations.)

On the one hand, if we ignore the anisotropy $\lambda$, the reduced temperature $t$ flows away from the unstable infrared fixed point $t=0$ to strong coupling with the beta function (see Refs. 50-53)

$$
\beta_{t}(t) \equiv \mathfrak{a} \frac{\partial t}{\partial \mathfrak{a}}=\sum_{n=1,2,3, \ldots}^{\infty} \beta_{n} t^{n}
$$

that vanishes to order $n=1$ and is given by the quadratic Casimir in the adjoint representation $C_{v}$

$$
\beta_{2}=k \times C_{v}=k \times N
$$

with $N=4$ to one-loop order $(n=2)$, up to the multiplicative factor $k$ that depends on the convention made for the normalization of the reduced temperature ( $k=1$ in Refs. 50-53). Correspondingly, as $t$ grows under this renormalization flow the correlation length $\xi(t)$ decreases,

$$
\xi(t) \sim \mathfrak{a} \exp \left(+\frac{1 /(k \times N)}{t}\right)
$$

with $N=4$. On the other hand, the mass scale $\lambda^{1 / 2}$ grows away from the Gaussian (unstable) fixed point $t=\lambda=0$ to leading order in the flow of the renormalization group according to

$$
\beta_{\lambda}(\lambda) \equiv \mathfrak{a} \frac{\partial \lambda}{\partial \mathfrak{a}}=2 \lambda
$$

The symmetry crossover is estimated from the condition $(\xi / \mathfrak{a})^{-2} \approx \lambda \ll 1$, i.e., ${ }^{54-56}$

$$
t \approx \frac{2 /(k \times N)}{\ln (c / \lambda)} \ll 1
$$

with $\lambda \ll c$ a positive constant of order one fixed by the shortdistance physics. Once Eq. (4.15) is fulfilled, it is the classical renormalization group flow in the unbroken subgroup of $\mathrm{U}(1)$ of SU(4) that takes over.

We conclude that the Kekulé mechanism can explain the experiment of Ref. 13 if (i) the phonon-induced Kekule instability can be boosted by the nearest-neighbor short-range Coulomb interaction that favors the Kekule instability so as to satisfy Eq. (3.2), (ii) the renormalized SU(4)-symmetric energy scale $J_{\mathrm{SU}(4)}$ [as opposed to the bare estimate Eq. (4.6)] that enters the reduced temperature in the NLSM satisfies

$$
T_{c} \approx \frac{1 /(k \times 4)}{\ln 50} \times J_{\mathrm{SU}(4)}[\mathrm{K}] \approx k^{-1} \times 0.12 \times J_{\mathrm{SU}(4)}[\mathrm{K}]
$$

since $\lambda$ is of order $\mathfrak{a} / \ell_{B} \sim 1 / 50$ for the relevant range of magnetic fields and if $c=1$ in Eq. (4.15). This estimate will be lowered by quantum fluctuations and by disorder (as described in Sec. II for the disorder).

\section{DISCUSSION}

In summary, we have argued that sufficiently large magnetic fields and sufficiently small temperatures (inside the shaded region of the phase diagram of Fig. 1) stabilize the Kekulé quasi-long-range order in graphene. This quasi-longrange order can be destroyed by the unbinding of vortices due to either thermal- or disorder-induced fluctuations. The role of the magnetic field $B$ is here twofold. First, $B$ enhances the density of states at the Dirac point, thereby favoring the Kekule distortion with the help of phonons. Second, a large $B$ prevents the disorder from filling a single-particle gap by suppressing the Landau degeneracy. The transition belongs to the 2D classical random phase XY model and is characterized by the deconfinement vortices for sufficiently small magnetic fields or sufficiently large temperatures (outside the shaded region of the phase diagram of Fig. 1). Each of these vortices, as we have shown here, bind a fraction of the electron charge for any magnetic field strength.

\section{ACKNOWLEDGMENTS}

This work was supported in part by DOE under Grant No. DEFG02-06ER46316 (C.-Y.H. and C.C.). C.M. acknowledges the kind hospitality of the Condensed Matter Theory Laboratory in RIKEN and the Condensed Matter Theory Visitor's Program at Boston University. We thank Y. Kopelevich, N. P. Ong, J. G. Checkelsky, S. Ryu, D.-H. Lee, K. Nomura, and A. Furusaki for useful and stimulating discussions.

\section{APPENDIX A: STIFFNESS}

In this appendix, we start from Hamiltonian (2.8) with the uniform magnetic field $B=\partial A_{y} / \partial_{x}-\partial A_{x} / \partial_{y}$ and with the uniform Kekulé order parameter $\Delta=\Delta_{0} e^{i \theta_{0}}$. We choose to work in the Landau gauge $\boldsymbol{A}=(-B y, 0)$. We are after the Kekulé phase stiffness.

To this end, we first need the eigenfunctions and eigenenergies of Hamiltonian (2.8). That the eigenenergies are given by Eq. (2.9) follows from the fact that the uniform Kekule order parameter anticommutes with the covariant derivative. Hence, any positive eigenenergy of $\mathcal{H}^{2}$ is nothing but the sum of two positive terms adding in quadrature. One is the eigenvalue of the square of the covariant derivatives in Eq. (2.8). The other is the eigenvalue of the square of the uniform Kekulé order parameter in Eq. (2.8). To obtain the (degenerate) eigenfunctions of any Landau level Eq. (2.9), we anticipate that any eigenfunction of Eq. (2.8) factorizes into the $x$-dependent phase $e^{i k x}$ times a four-component spinor that depends solely on $y$ through the dimensionless coordinate $\xi=\left(y / \ell_{B}\right)+k \ell_{B}$. As it does for the conventional (nonrelativistics) Landau levels, the wave number $k$ encodes a degeneracy of the spectrum [Eq. (2.9)] that scales with the area of the system. An additional (relativistic) source of degeneracy of the spectrum [Eq. (2.9)] arises because of the dimensionality four of the Dirac matrices. This finite degen- 
eracy in the sole presence of a uniform magnetic field is selectively lifted by the uniform Kekulé order parameter. Indeed, the single-particle eigenstates with $N=0$ are nondegenerate for each wave number $k$ as a result of the Kekulé instability and given by

$$
\Psi_{k, 0, \pm}(x, \xi)=\frac{e^{i k x}}{\sqrt{2}}\left(\begin{array}{c}
\varphi_{0}(\xi) \\
0 \\
\pm \varphi_{0}(\xi) e^{-i \theta_{0}} \\
0
\end{array}\right)
$$

On the other hand, for any wave number $k$, positive integer $N=1,2, \ldots$, and sign \pm , the pair of orthonormal eigenfunctions

and

$$
\widetilde{\Psi}_{k, N, \pm}(x, \xi)=\frac{e^{i k x}}{\sqrt{2}}\left(\begin{array}{c}
\frac{\Delta_{0}}{\varepsilon_{N,+}} \varphi_{N}(\xi) \\
0 \\
\pm \varphi_{N}(\xi) e^{-i \theta_{0}} \\
\frac{-\hbar \omega_{\mathrm{c}} \sqrt{N}}{\varepsilon_{N,+}} \varphi_{N-1}(\xi) e^{-i \theta_{0}}
\end{array}\right)
$$

remain twofold degenerate in spite of the Kekulé instability. Here, $\varphi_{N}$ with $N=0,1,2, \ldots$ are the orthonormal eigenfunctions of the one-dimensional quantum harmonic oscillator.

Next, the Kekule phase stiffness can be computed by adding all the changes in the negative single-particle energy levels [Eq. (2.9)] up to the Fermi level $\mu=0$ that are induced by the twist $\Delta \rightarrow \Delta \exp (i q \cdot r)$ in the Kekule order parameter.

Before doing this, it is convenient to gauge out the spatial dependence of the Kekule order parameter with the help of the pure axial gauge transformation $\Psi^{\prime} \equiv U \Psi$, where $U \equiv e^{-i \boldsymbol{q} \cdot \boldsymbol{r} \gamma_{5} / 2}$. Under this transformation, the Hamiltonian becomes $\mathcal{H}^{\prime}=\mathcal{H}+\mathcal{V}$, where $\mathcal{H}$ is given by Eq. (2.8) with the Landau spectrum Eq. (2.9) and $\mathcal{V}=v_{\mathrm{F}} \boldsymbol{q} \cdot \boldsymbol{\alpha} \gamma_{5} / 2$

We treat $\mathcal{V}$ as a perturbation, assume that $\hbar v_{\mathrm{F}}|\boldsymbol{q}|$ is small compared to $\Delta_{0}$, and compute the change in the spectrum of $\mathcal{H}$ induced by $\mathcal{V}$ up to second order in degenerate perturbation theory. Because the perturbation $\mathcal{V}$ does not couple eigenstates with different wave number $k$, the degeneracy of each unperturbed Landau level Eq. (2.9) is conserved. Furthermore, these second-order shifts $\varepsilon_{k, N, \pm}^{(2)}$ cancel pairwise for all twofold degenerate levels: $\varepsilon_{k, N,+}^{(2)}+\varepsilon_{k, N,-}^{(2)}=0$ when
$N=1,2, \ldots$ Hence at half-filling $(\mu=0)$, the second order shifts from all states with $N=1,2, \ldots$ cancel pairwise, leaving the unpaired $N=0$ states as sole contributors to the total energy shift

$$
\delta E=2 \times \sum_{k} \varepsilon_{k, 0,-}^{(2)},
$$

where the factor of 2 accounts for the spin-1/2 degeneracy, and the second-order correction,

$$
\varepsilon_{k, 0,-}^{(2)}=v_{\mathrm{F}}^{2}|\boldsymbol{q}|^{2} \frac{\Delta_{0}}{2 \omega_{\mathrm{c}}^{2}}=\frac{\ell_{B}^{2}}{4}|\boldsymbol{q}|^{2} \Delta_{0}
$$

are independent of $k$. The total-energy shift per unit area $\delta E / \mathcal{A}$ can be obtained by accounting for the density of states per area and per spin $1 /\left(2 \pi \ell_{B}^{2}\right)$ at the unperturbed energy $-\Delta_{0}$, i.e.,

$$
\frac{\delta E}{\mathcal{A}}=\frac{\Delta_{0}}{4 \pi}|\boldsymbol{q}|^{2} .
$$

If we define the stiffness by

$$
\frac{\delta E}{\mathcal{A}} \equiv \frac{J}{2}|\boldsymbol{q}|^{2}
$$

we deduce that

$$
J=\frac{\Delta_{0}}{2 \pi}
$$

Because the self-consistent Kekulé gap $\Delta_{0}$ induced by a magnetic field and an electron-phonon interaction scales linearly in $B$, the spinstiffness also scales linearly with $B$.

\section{APPENDIX B: ZERO MODES}

The zero modes of the Dirac Hamiltonian (2.8), when the Kekule order parameter $\Delta$ is defective in that it carries a vortex of vorticity \pm 1 , can be found analytically and shown to exist for all strengths of the applied magnetic field. In this appendix, we choose to work in the symmetric gauge $\boldsymbol{A}$ $=\frac{B}{2}(-y, x)$, which we write as the complex number $A$ $=i B z / 2$ with $z \equiv x+i y$, and seek the solutions (zero modes) to 


$$
\left(\begin{array}{cccc}
0 & \hbar v_{\mathrm{F}}\left(-2 i \partial_{z}+\frac{i \bar{z} \ell_{B}^{2}}{2}\right) & \Delta & 0 \\
\hbar v_{\mathrm{F}}\left(-2 i \partial_{\bar{z}}-\frac{i z \ell_{B}^{2}}{2}\right) & 0 & 0 & \Delta \\
\bar{\Delta} & 0 & 0 & \hbar v_{\mathrm{F}}\left(+2 i \partial_{z}-\frac{i \bar{z} \ell_{B}^{2}}{2}\right) \\
0 & \bar{\Delta} & \hbar v_{\mathrm{F}}\left(+2 i \partial_{\bar{z}}+\frac{i z \ell_{B}^{2}}{2}\right) & 0
\end{array}\right) \Psi=0 .
$$

The spinor

$$
\Psi_{\mathrm{A}}(\boldsymbol{r})=\left(\begin{array}{c}
0 \\
u(\boldsymbol{r}) \\
v(\boldsymbol{r}) \\
0
\end{array}\right)
$$

is a zero mode supported on sublattice A if

$$
\begin{gathered}
\hbar v_{\mathrm{F}}\left(-2 i \partial_{z}+\frac{i \bar{z} \ell_{B}^{2}}{2}\right) u+\Delta v=0, \\
\bar{\Delta} u+\hbar v_{\mathrm{F}}\left(2 i \partial_{\bar{z}}+\frac{i z \ell_{B}^{2}}{2}\right) v=0,
\end{gathered}
$$

and $u$ and $v$ are normalizable. Switching to polar coordinates and considering an antivortex (vorticity $n_{\mathrm{v}}=-1$ ) for concreteness, i.e., $\Delta=\Delta(\rho, \theta)=\Delta_{0} e^{i \theta_{0}} e^{-i \theta}$, the conditions on the components $u$ and $v$ become

$$
\begin{gathered}
-i \hbar v_{\mathrm{F}}\left(\partial_{\rho}-\frac{i}{\rho} \partial_{\theta}-\frac{\rho}{2 \ell_{B}^{2}}\right) u+\Delta_{0} e^{i \theta_{0}} v=0, \\
\Delta_{0} e^{-i \theta_{0}} u+i \hbar v_{\mathrm{F}}\left(\partial_{\rho}+\frac{i}{\rho} \partial_{\theta}+\frac{\rho}{2 \ell_{B}^{2}}\right) v=0 .
\end{gathered}
$$

The homogeneous system [Eq. (B3)] of first-order partial differential equations admits $\theta$-independent solutions $u(\rho)$ and $v(\rho)$. Indeed, solving for $u$ in terms of $v$ in Eq. (B3b) gives

$$
u=-i \hbar v_{\mathrm{F}} \Delta_{0}^{-1} e^{i \theta_{0}}\left(\partial_{\rho}+\frac{\rho}{2 \ell_{B}^{2}}\right) v,
$$

and, after substitution into Eq. (B3a),

$$
-\left(\ell_{B} \partial_{\rho}\right)^{2} v+\frac{1}{4}\left(\frac{\rho}{\ell_{B}}\right)^{2} v=-\left(\frac{1}{2}+p\right) v,
$$

where

$$
p=\left(\frac{\ell_{B} \Delta_{0}}{\hbar v_{\mathrm{F}}}\right)^{2}=\frac{2 \Delta_{0}^{2}}{\left(\hbar \omega_{\mathrm{c}}\right)^{2}} .
$$

Equation (B4b) would be identical to the second-order differential equation describing a 1D quantum harmonic oscillator were it not for the fact that the radial coordinate $\rho$ is always positive. Equation (B4b) thus admits normalizable solutions unavailable to the $1 \mathrm{D}$ quantum harmonic oscillator, i.e., any normalizable solution on the half line that blows up when $\rho \rightarrow-\infty$ is here allowed. The solutions of Eq. (B4b) for $\rho>0$ are parabolic cylinder functions $D_{-p}\left(\rho / \ell_{B}\right),{ }^{37}$ parametrized by the dimensionless ratio $p$. We conclude, when the Kekulé order parameter admits an antivortex of charge $n_{\mathrm{v}}=-1$, that a normalizable zero mode of Eq. (B1) can be expressed in terms $u$ and $v$ satisfying Eqs. (2.21a) and (2.21b). The solution for the case of vorticity $n_{\mathrm{v}}=+1$ is analogous, but with a zero mode supported on sublattice B.
${ }^{1}$ R. B. Laughlin, Phys. Rev. Lett. 50, 1395 (1983).

${ }^{2}$ L. Saminadayar, D. C. Glattli, Y. Jin, and B. Etienne, Phys. Rev. Lett. 79, 2526 (1997).

${ }^{3}$ R. De Picciotto, M. Reznikov, M. Heiblum, V. Umansky, G. Bunin, and D. Mahalu, Nature (London) 389, 162 (1997).

${ }^{4}$ J. Martin, S. Ilani, B. Verdene, J. Smet, V. Umansky, D. Mahalu, D. Schuh, G. Abstreiter, and A. Yacoby, Science 305, 980 (2004).

${ }^{5}$ D. S. Rokhsar and S. A. Kivelson, Phys. Rev. Lett. 61, 2376 (1988).

${ }^{6}$ R. Moessner and S. L. Sondhi, Phys. Rev. Lett. 86, 1881 (2001).
${ }^{7}$ C.-Y. Hou, C. Chamon, and C. Mudry, Phys. Rev. Lett. 98, 186809 (2007).

${ }^{8}$ R. Jackiw and C. Rebbi, Phys. Rev. D 13, 3398 (1976).

${ }^{9}$ W. P. Su, J. R. Schrieffer, and A. J. Heeger, Phys. Rev. Lett. 42, 1698 (1979).

${ }^{10}$ R. Jackiw and S.-Y. Pi, Phys. Rev. Lett. 98, 266402 (2007).

${ }^{11}$ C. Chamon, Phys. Rev. B 62, 2806 (2000).

12 J. G. Checkelsky, L. Li, and N. P. Ong, Phys. Rev. Lett. 100, 206801 (2008).

${ }^{13}$ J. G. Checkelsky, L. Li, and N. P. Ong, Phys. Rev. B 79, 115434 (2009). 
${ }^{14}$ P. R. Wallace, Phys. Rev. 71, 622 (1947).

${ }^{15}$ J. W. McClure, Phys. Rev. 104, 666 (1956).

${ }^{16}$ S. Deser, R. Jackiw, and S. Templeton, Ann. Phys. (N.Y.) 140, 372 (1982).

${ }^{17}$ A. J. Niemi and G. W. Semenoff, Phys. Rev. Lett. 51, 2077 (1983).

${ }^{18}$ A. N. Redlich, Phys. Rev. Lett. 52, 18 (1984).

${ }^{19}$ K. Ishikawa, Phys. Rev. Lett. 53, 1615 (1984).

${ }^{20}$ V. P. Gusynin and S. G. Sharapov, Phys. Rev. Lett. 95, 146801 (2005).

${ }^{21}$ K. S. Novoselov, A. K. Geim, S. V. Morozov, D. Jiang, M. I. Katsnelson, I. V. Grigorieva, S. V. Dubonos, and A. A. Firsov, Nature (London) 438, 197 (2005).

${ }^{22}$ Y. Zhang, Yan-Wen Tan, Horst L. Stormer, and Philip Kim, Nature (London) 438, 201 (2005).

${ }^{23}$ Y. Zhang, Z. Jiang, J. P. Small, M. S. Purewal, Y.-W. Tan, M. Fazlollahi, J. D. Chudow, J. A. Jaszczak, H. L. Stormer, and P. Kim, Phys. Rev. Lett. 96, 136806 (2006).

${ }^{24}$ Z. Jiang, Y. Zhang, H. L. Stormer, and P. Kim, Phys. Rev. Lett. 99, 106802 (2007).

${ }^{25}$ A. H. Castro Neto, F. Guinea, N. M. Peres, K. S. Novoselov, and A. K. Geim, Rev. Mod. Phys. 81, 109 (2009).

${ }^{26}$ J. M. Kosterlitz and D. J. Thouless, J. Phys. C 6, 1181 (1973); J. M. Kosterlitz, ibid. 7, 1046 (1974).

${ }^{27}$ For example, a sample with $B_{c}=32.1 \mathrm{~T}$ yields $\Delta_{\mathrm{a}}=12.9 \mathrm{~K}$ at $B=30 \mathrm{~T}$ and $\Delta_{\mathrm{a}}=14.7 \mathrm{~K}$ at $B=31.2 \mathrm{~T}$.

${ }^{28}$ K. Nomura, S. Ryu, and D.-H. Lee, Phys. Rev. Lett. 103, 216801 (2009).

${ }^{29}$ S. Ryu, C. Mudry, C.-Y. Hou, and C. Chamon, Phys. Rev. B 80, 205319 (2009).

${ }^{30}$ H. Ajiki and T. Ando, J. Phys. Soc. Jpn. 64, 260 (1995).

${ }^{31}$ J.-N. Fuchs and P. Lederer, Phys. Rev. Lett. 98, 016803 (2007).

${ }^{32}$ E. Shimshoni, H. A. Fertig, and G. V. Pai, Phys. Rev. Lett. 102, 206408 (2009).

${ }^{33}$ S. Sachdev, Phys. Rev. B 59, 14054 (1999).

${ }^{34}$ For a review, see S. E. Korshunov, Usp. Fiz. Nauk 176, 233 (2006).
${ }^{35}$ C. Mudry and X.-G. Wen, Nucl. Phys. B 549, 613 (1999).

${ }^{36}$ D. Carpentier and P. Le Doussal, Nucl. Phys. B 588, 565 (2000).

${ }^{37}$ I. S. Gradshteyn and I. M. Ryzhik, Table of Integrals, Series, and Products, 6th ed. (Academic, London, 2000), pp. 1018-1023.

${ }^{38}$ C. Chamon, C.-Y. Hou, R. Jackiw, C. Mudry, S.-Y. Pi, and A. P. Schnyder, Phys. Rev. Lett. 100, 110405 (2008).

${ }^{39}$ C. Chamon, C.-Y. Hou, R. Jackiw, C. Mudry, S.-Y. Pi, and G. Semenoff, Phys. Rev. B 77, 235431 (2008).

${ }^{40}$ K. Nomura and A. H. MacDonald, Phys. Rev. Lett. 96, 256602 (2006).

${ }^{41}$ J. Alicea and M. P. A. Fisher, Phys. Rev. B 74, 075422 (2006).

${ }^{42}$ M. O. Goerbig, R. Moessner, and B. Doucot, Phys. Rev. B 74, 161407(R) (2006).

${ }^{43}$ X. G. Wen and A. Zee, Phys. Rev. Lett. 69, 1811 (1992); Phys. Rev. B 47, 2265 (1993).

${ }^{44}$ K. Moon, H. Mori, Kun Yang, S. M. Girvin, A. H. MacDonald, L. Zheng, D. Yoshioka, and Shou-Cheng Zhang, Phys. Rev. B 51, 5138 (1995).

${ }^{45}$ K. Yang, K. Moon, L. Belkhir, H. Mori, S. M. Girvin, A. H. MacDonald, L. Zheng, and D. Yoshioka, Phys. Rev. B 54, 11644 (1996)

${ }^{46}$ H. Eichenherr, Nucl. Phys. B 146, 215 (1978).

${ }^{47}$ A. d'Adda, M. Luescher, and P. di Vecchia, Nucl. Phys. B 146, 63 (1978).

${ }^{48}$ V. L. Golo and A. M. Perelomov, Phys. Lett. B 79, 112 (1978).

${ }^{49}$ A. M. Polyakov, Gauge Fields and Strings (Harwood Academic, London, 1987).

${ }^{50}$ E. Brézin, S. Hikami, and J. Zinn-Justin, Nucl. Phys. B 165, 528 (1980).

${ }^{51}$ S. Hikami, Phys. Lett. B 98, 208 (1981).

${ }^{52}$ S. Hikami, Nucl. Phys. B 215, 555 (1983).

${ }^{53}$ F. Wegner, Nucl. Phys. B 316, 663 (1989).

${ }^{54}$ R. A. Pelcovits and D. R. Nelson, Phys. Lett. A 57, 23 (1976).

${ }^{55}$ S. B. Khokhlachev, Zh. Eksp. Teor. Fiz. 70, 265 (1976) [Sov. Phys. JETP 43, 137 (1976)].

${ }^{56}$ S. Hikami and T. Tsuneto, Prog. Theor. Phys. 63, 387 (1980). 